\title{
Robotic surgery of gallbladder cancer
}

\author{
Andrea Belli', Renato Patrone², Vittorio Albino', Maddalena Leongito', Mauro Piccirillo', Vincenza \\ Granata $^{3}$, Gilda Pasta4, Raffaele Palaia', Francesco Izzo'1
}

\begin{abstract}
'Department of Abdominal Oncology, Division of Hepatobiliary Surgical Oncology, Istituto Nazionale Tumori, Fondazione G. Pascale, IRCCS, Napoli 80131, Italy.

2Department of Cardiothoracic Sciences, Division of General and Oncologic Surgery, University of Campania "Luigi Vanvitelli", School of Medicine, Naples 80131, Italy.

${ }^{3}$ Division of Radiology, Istituto Nazionale Tumori, Fondazione G. Pascale, IRCCS, Napoli 80131, Italy.

${ }^{4}$ Division of Anesthesia, Pain medicine and Supportive Care, Istituto Nazionale Tumori, Fondazione G. Pascale, IRCCS, Napoli 80131, Italy.
\end{abstract}

Correspondence to: Dr. Andrea Belli, Department of Abdominal Oncology, Division of Hepatobiliary Surgical Oncology, Istituto Nazionale Tumori, IRCCS, Fondazione G. Pascale, Via Mariano Semmola, Napoli 80131, Italy. E-mail: a.belli@istitutotumori.na.it

How to cite this article: Belli A, Patrone R, Albino V, Leongito M, Piccirillo M, Granata V, Pasta G, Palaia R, Izzo F. Robotic surgery of gallbladder cancer. Mini-invasive Surg 2020;4:77. http://dx.doi.org/10.20517/2574-1225.2020.70

Received: 6 Jul 2020 First Decision: 21 Aug 2020 Revised: 31 Aug 2020 Accepted: 14 Sep 2020 Published: 6 Nov 2020

Academic Editor: Giulio Belli Copy Editor: Cai-Hong Wang Production Editor: Jing Yu

\begin{abstract}
Aim: The aim of this study was to describe our technique for the surgical treatment of clinically suspected or incidentally diagnosed gallbladder cancer (GBC) and to report the outcomes of our experience.

Methods: This is a retrospective observational study including consecutive patients operated by a robotic approach for the surgical treatment of clinically suspected or incidentally diagnosed GBC (with the intent of radical reresection after index cholecystectomy) performed between January 2017 and December 2019. Clinical outcomes and technical details related to the robotic approach were analyzed.
\end{abstract}

Results: During the study period, 8 patients underwent robotic radical cholecystectomy with lymphadenectomy and atypical resection of segments IVb-V. No conversion or major complications occurred intraoperatively. All patients underwent a radical resection. There were one Clavien-Dindo grade II and one grade IIIb complication. Median hospital stay was 6 days (range 5-11). At a median follow-up of 17.5 months (range 29.3-7.3), all patients are alive and free from disease except one who had peritoneal recurrence and underwent chemotherapy. No trocar site recurrence was observed.

Conclusion: The present study describes a standardized step-by-step robotic technique for the surgical treatment of GBC and demonstrates the feasibility and safety of the robotic approach. More data and multicentre series are

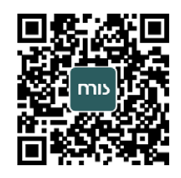


needed to confirm our results and to assess the oncologic outcomes of the robotic approach.

Keywords: Gallbladder cancer, robotic surgery, radical cholecystectomy, incidental gallbladder cancer, lymphadenectomy, minimally invasive surgery

\section{INTRODUCTION}

Minimally invasive approaches are gradually becoming a standard of care in abdominal surgical oncology. Several high-quality studies including randomized controlled trials demonstrated non-inferiority in terms of oncologic outcomes of the laparoscopic approach for the treatment of colorectal and gastric cancer and confirmed the advantages of minimal invasiveness in terms of perioperative outcomes and length of postoperative stay $^{[1-3]}$. The feasibility, safety and oncologic non-inferiority of laparoscopic liver resection have already been established as well as the advantages of the minimally invasive approach in terms of intraoperative bleeding and short-term outcomes ${ }^{[4-7]}$. The minimally invasive approach to liver neoplasms is more and more applied worldwide and is becoming a routine approach in dedicated centres in selected patients for the surgical treatment of colorectal liver metastases and hepatocellular carcinoma $a^{[4-7]}$. However, there is a strong reluctance to the adoption of the minimally invasive approach for the treatment of gallbladder cancer (GBC), which is one of the most aggressive cancers of the biliary tract and is generally associated with a poor prognosis. This scepticism is historically related to the fear of tumour dissemination due to bile spillage, tumour manipulation during laparoscopy, possible tumour peritoneal implantation due to the pneumoperitoneum as well as to technical difficulties related to liver resection and to the achievement of an adequate clearance of lymph nodes. Recently, some reports have advocated the minimally invasive surgical treatment of clinically suspected or incidentally diagnosed GBC, highlighting the feasibility and apparent safety of this approach ${ }^{[8-13]}$. Nevertheless, only few authors have reported on the feasibility and outcomes of the surgical treatment of GBC by a robotic approach, which has the potential to facilitate, by the articulated instrumentations and magnified $3 \mathrm{D}$ view, the accomplishment of the procedure and the locoregional lymphadenectomy needed to obtain a radical resection and an accurate staging of the resected patients. The aim of this study was to report the outcomes of our initial experience with the robotic treatment of clinically suspected or incidentally diagnosed GBC and to highlight the technical details related to the robotic approach.

\section{METHODS}

This was a retrospective observational study including consecutive patients operated by a robotic approach for the surgical treatment of clinically suspected or incidentally diagnosed GBC (with the intent of radical re-resection after index cholecystectomy) at the National Cancer Institute - G. Pascale - IRCCS of Naples, Italy. Patients without relevant comorbidities precluding a minimally invasive approach were considered for robotic resection in case of the following.

(1) A suspected preoperative diagnosis of GBC without massive liver involvement and/or suspicion of bile duct invasion ( $\mathrm{T}$ stage $>1 \mathrm{~b}$ and $<\mathrm{T} 4$ ) and no suspicion of peritoneal carcinomatosis.

(2) Patients already submitted to cholecystectomy for presumed benign disease and an incidental diagnosis of GBC ( $\mathrm{T}$ stage $>1 \mathrm{~b}$ ) without massive liver involvement and/or suspicion of bile duct invasion and no suspicion of peritoneal carcinomatosis.

The preoperative staging protocol included standard blood tests including carcinoembryonic antigen, carbohydrate antigen (CA) 19.9 and CA 125, a total-body CT scan and an abdominal MRI. An FDG-PET was used selectively in case of suspected advanced disease. Informed consent was obtained and patients 
who refused the minimally invasive approach were offered a standard open operation. In case of a previous cholecystectomy, operative notes of the index gallbladder resection were carefully reviewed, and the surgeons who performed the operations were contacted whenever possible. Clinicopathological features of the patients were prospectively recorded in a dedicated electronic database and included age, sex, American Society of Anesthesiology score, previous abdominal surgery, tumour dimensions and $\mathrm{T}$ stage at preoperative imaging. Intraoperative variables analysed included operative time (divided into robotic system docking time and skin incision to skin closure time), occurrence and type of intraoperative complications, need and cause of conversion to laparoscopy or to laparotomy, blood loss, need for intraoperative transfusions, and occurrence of bile spillage. Surgery-related mortality was defined as death occurring during hospital admission or within 90 days of the operation. Occurrence of postoperative complications with 90 days were graded by the Clavien-Dindo classification ${ }^{[14]}$. Specific morbidity related to lymphadenectomy was assessed in terms of biliary or vascular injuries, postoperative pancreatitis, bleeding and the occurrence of lymphatic fistula (defined as the presence of triglycerides in drain fluid $>110 \mathrm{mg} / \mathrm{dL}$ ), bile leaks were graded by the definition of the International Study Group of Liver Surgery ${ }^{[15]}$. Postoperative recorded variables included length of hospital stay, radicality of the resection (R1 resection defined as any microscopically positive margin or a cancer-free margin $<1 \mathrm{~mm}$ ), $\mathrm{T}$ stage and number of retrieved and positive nodes at final pathology, M status, need for and accomplishment of postoperative chemotherapy. Oncologic follow-up included blood tests, tumour markers and a total-body CT scan at 40 days after the operation and at 3 and 6 months thereafter. Type and location of recurrence were prospectively recorded as well as the type of treatment administered when needed.

\section{Surgical technique}

Step 1

The patient is placed supine and legs apart in a slight reverse Trendelenburg and tilted to the left. A periumbilical incision is made, and the pneumoperitoneum is created by the open technique. Once the abdomen is insufflated, a staging laparoscopy is performed to exclude any sign of peritoneal carcinomatosis or diffuse hepatic involvement. In case of no contraindications to the procedure, 3 additional robotic trocars and one service 12- $\mathrm{mm}$ port for the assistant surgeon are inserted under direct view [Figure 1], and the robotic da Vinci Xi Surgical System ${ }^{\circledR}$ (Intuitive Surgical Inc. Sunnyvale, CA, USA) is docked. The first surgeon is at the robotic console while the assistant surgeon stands between the patient's legs.

\section{Step 2}

Intraoperative liver ultrasound is performed to assess the presence of liver invasion and its depth, and to exclude any other intrahepatic metastasis as well. In case of re-intervention after a previous cholecystectomy, any fatty or omental adhesions to the gallbladder bed are left in place with the aim of being resected en bloc with the liver resection specimen. The right colonic flexure is then mobilized and a wide Kocker manoeuvre is carried out to greatly expose the inferior vena cava and aorta. The lymph nodes of station 16 (aortocaval nodes) are excised from the gonadic vein caudally up to the left renal vein cranially by the aid of robotic scissors and robotic Maryland bipolar forceps and sent for frozen section analysis [Figure 2]. In case of node involvement the procedure is abandoned.

\section{Step 3}

Retropancreatic lymph nodes (i.e., station 13) are carefully excised avoiding injuries to the duodenum or the pancreatic head. This manoeuvre is facilitated by the $3 \mathrm{D}$ high-definition view of the surgical area and by the articulated robotic scissors and Maryland bipolar forceps [Figure 3]. The lymphadenectomy continues on the right lateral border of the hepatic pedicle (i.e., station 12). In case of re-intervention for revision post-cholecystectomy surgery at this level, even in the presence of inflammatory tissue, which can make the dissection difficult, an effort is made to isolate and resect the stump of the clipped cystic duct for frozen section analysis. In case of no previous cholecystectomy, Calot's triangle is dissected and the cystic duct 


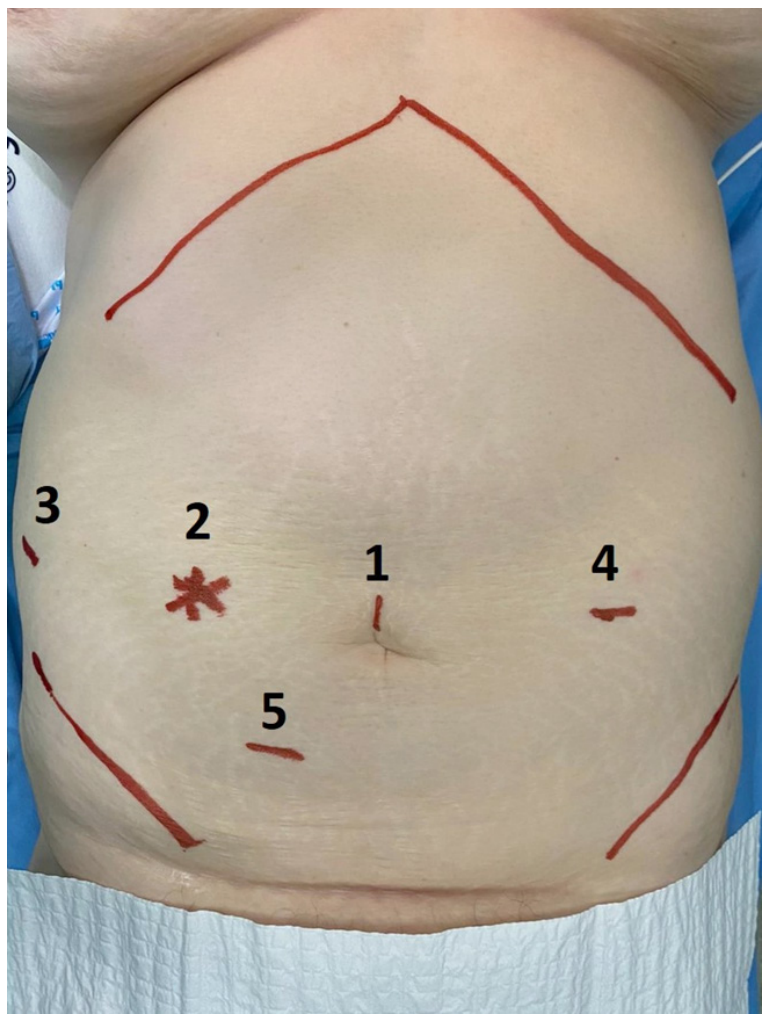

Figure 1. Sites for placement of trocars: (1) initial incision, robotic instrument and site for specimen extraction; (2) robotic optical system; (3) robotic instrument; (4) robotic instrument; and (5) laparoscopic port for assistant surgeon

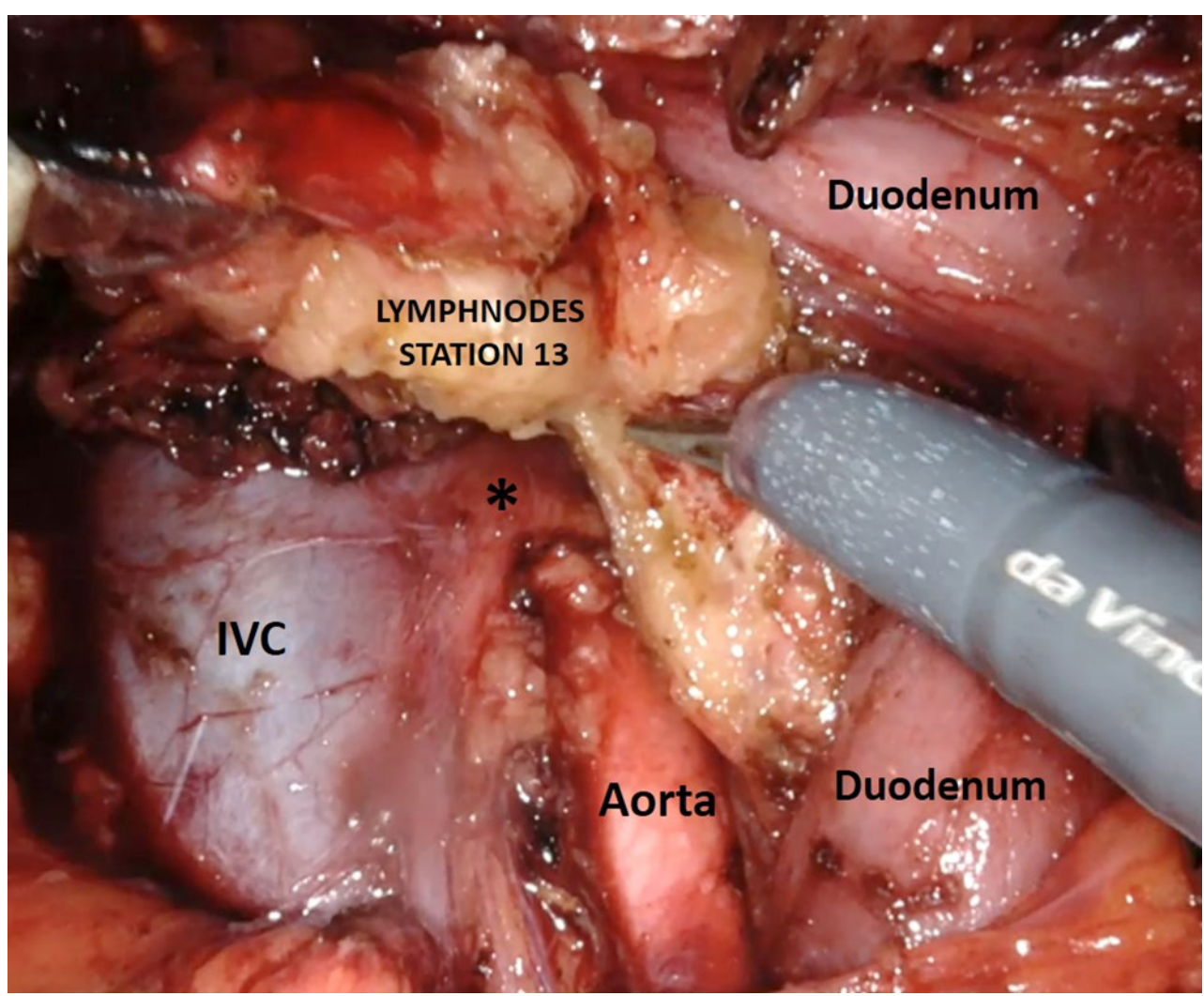

Figure 2. Intraoperative view of robotic retropancreatic lymphadenectomy. *Left renal vein. IVC: inferior vena cava 


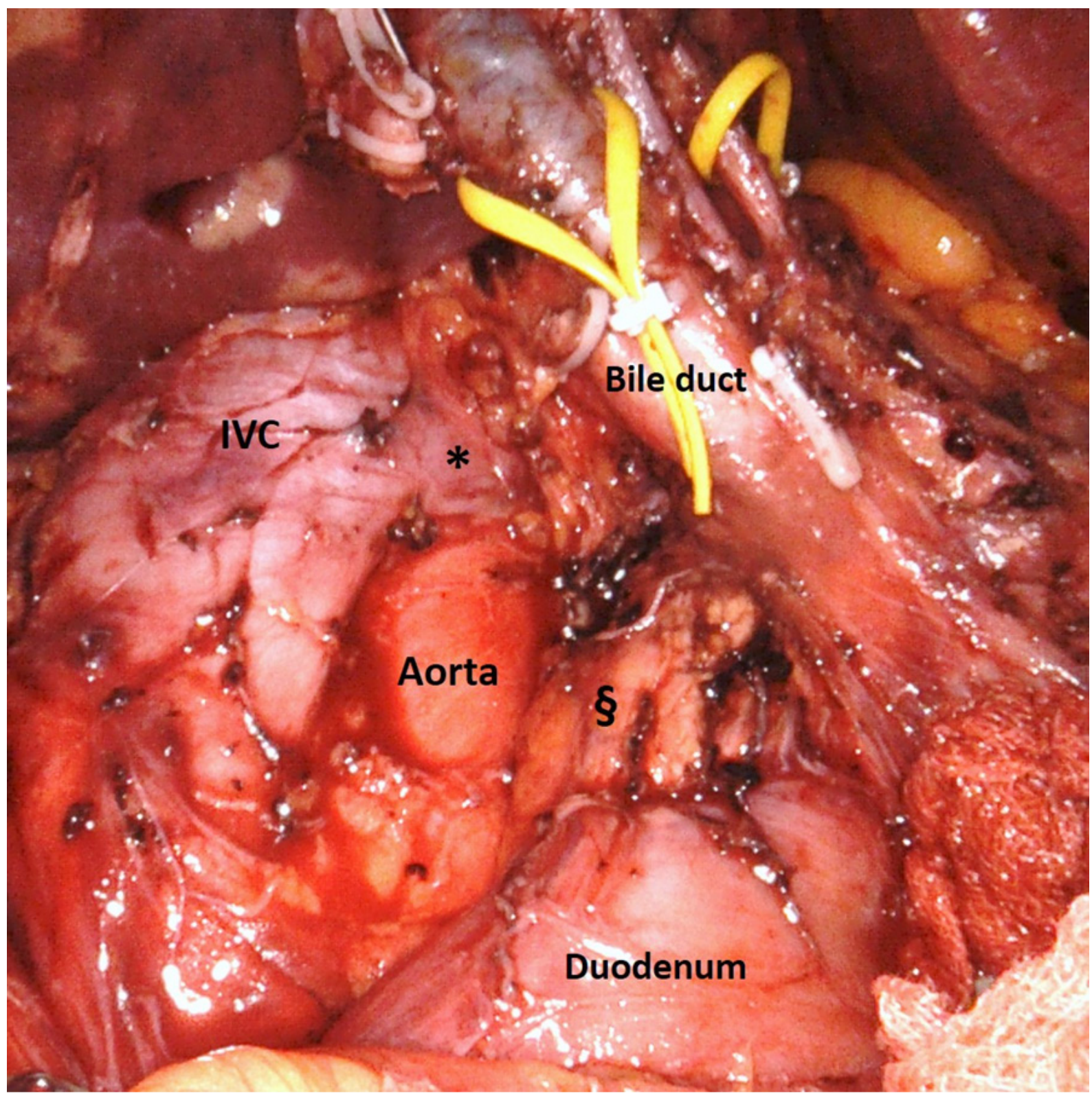

Figure 3. Final view after robotic lymphadenectomy. ${ }^{5}$ Posterior aspect of pancreatic head; *left renal vein. IVC: inferior vena cava

and cystic artery are isolated and sectioned between clips. Retroportal soft tissue and the left side of the hepatoduodenal ligament are then dissected, and the structures of the portal triad skeletonized and hung on vessel loops [Figure 4]. This manoeuvre allows a complete clearance of station 9. The lymphadenectomy is completed by dissecting the proper and common hepatic artery (i.e., station 8), proceeding from the right to the left. Lymph nodes are finally collected ideally en-bloc and put in a plastic bag.

\section{Step 4}

The intended liver resection plane, which is tailored to the presence and the extent of tumour invasion into the parenchyma (a minimum of $2 \mathrm{~cm}$ in width wedge resection of segments $\mathrm{IVb}-\mathrm{V}$ ), is marked on the Glissonian surface with monopolar robotic scissors, and an ultrasound repeated to check the margin width and liver anatomy. Liver resection is then carried out (en bloc with the gallbladder when present) with the aid of moist robotic Maryland bipolar forceps and robotic vessel sealer. Any relevant biliary or 


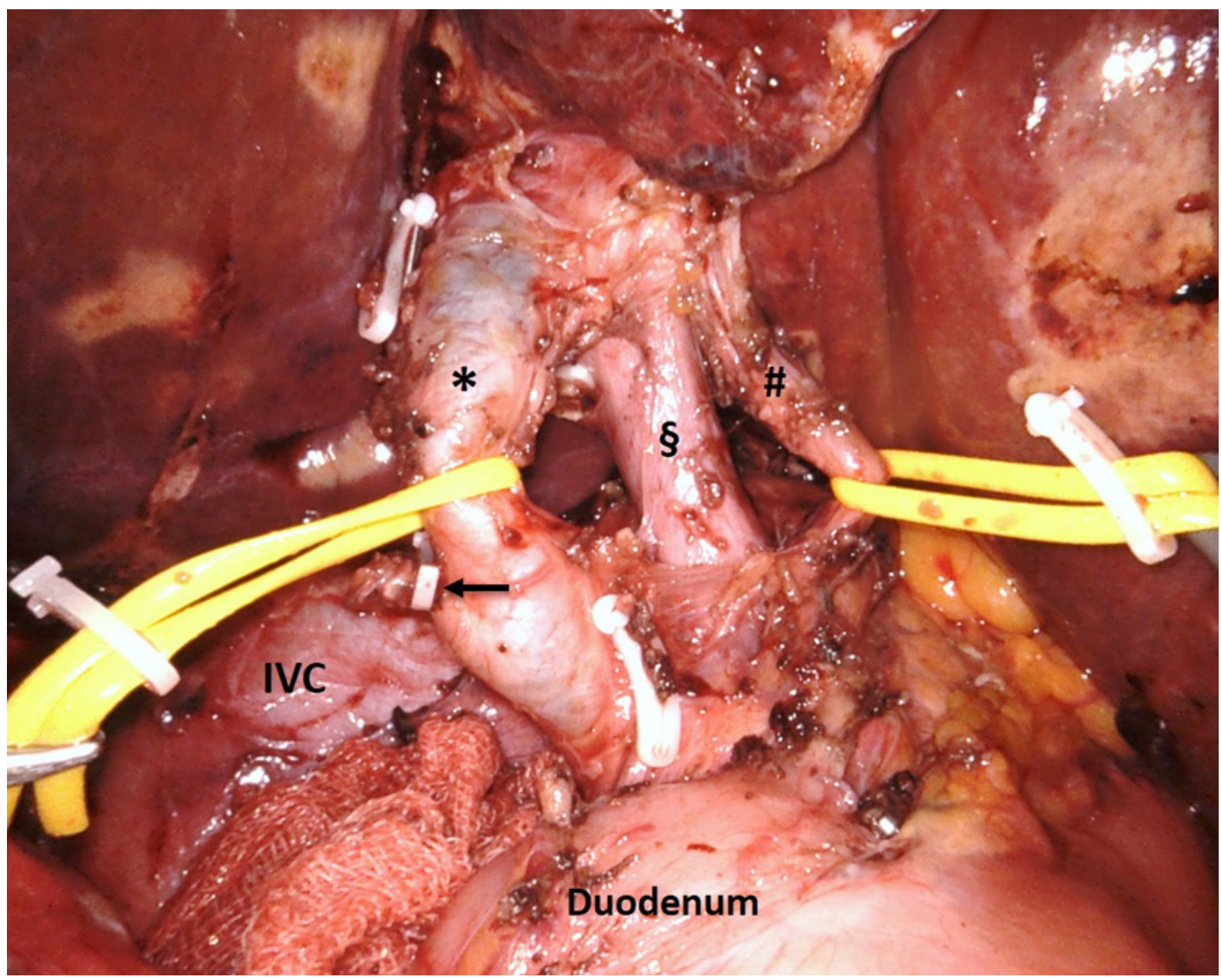

Figure 4. Final view after robotic lymphadectomy of the hepatoduodenal ligament. ${ }^{\star}$ Bile duct; sportal vein; " hepatic artery; black arrow: stump of the cystic duct. IVC: inferior vena cava

vascular structures are carefully isolated and clipped to avoid postoperative bleeding or bile leaks. Once the resection is completed, the raw liver surface is further cauterized with robotic Maryland bipolar forceps [Figure 5] and haemostatic agents or patch can be applied on the resection bed at the surgeon's discretion. The liver specimen is placed in a plastic bag and a drain is placed under the cut liver with the tip under the hepatoduodenal ligament. Surgical specimens are extracted in plastic bags via a slightly enlarged periumbilical incision and the pneumoperitoneum aspired.

\section{RESULTS}

Between January 2017 and December 2019, eight patients were operated by a robotic approach for a suspected preoperative diagnosis of GBC or for radicalisation after an incidental diagnosis of GBC at the National Cancer Institute - IRCCS Fondazione G. Pascale of Naples, Italy. Patient characteristics are given in Table 1. Four patients were female and four were male. The mean age of the patients was 70 years (range 42-89 years). Five patients were submitted to robotic radical cholecystectomy for suspected GBC, while three patients were submitted to radical revision surgery for an incidental diagnosis of GBC after index cholecystectomy. Pre- and postoperative tumour characteristics are described in Table 2. All patients were submitted to an extended lymphadenectomy plus a liver resection of the gallbladder bed (ultrasoundguided wedge resection of segment IVb-V with a minimum of $2-3 \mathrm{~cm}$ tumour-free margin). In all cases, the operation was successfully concluded by a robotic approach without the need for conversion to laparoscopic 
Table 1. Patient characteristics

\begin{tabular}{ll}
\hline Patients & $\boldsymbol{n} /$ total (\%) \\
\hline Gender & \\
Male & $4 / 8(50 \%)$ \\
Female & $4 / 8(50 \%)$ \\
Age & \\
Years & $70($ range $42-89)$ \\
ASA & \\
1 & $0 / 8(0 \%)$ \\
2 & $2 / 8(25 \%)$ \\
3 & $6 / 8(75 \%)$ \\
4 & $0 / 8(0 \%)$ \\
Previous cholecystectomy & \\
Yes & $3 / 8(37.5 \%)$ \\
No & $5 / 8(62.5 \%)$ \\
Previous abdominal surgery & \\
Yes & $5 / 8(62.5 \%)$ \\
No & $3 / 8(37.5 \%)$ \\
\hline
\end{tabular}

ASA: American Society of Anesthesiology

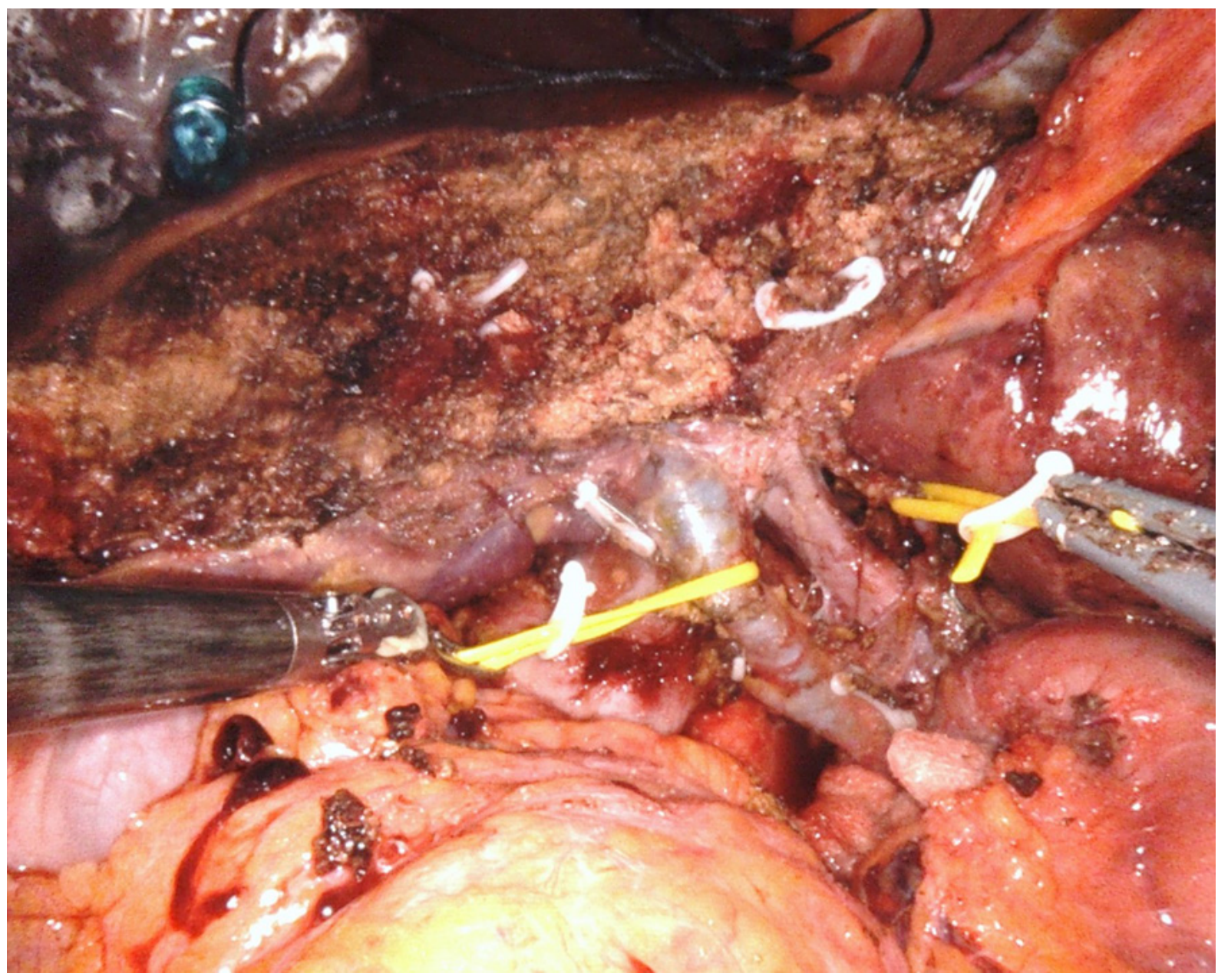

Figure 5. Final intraoperative view at the end of the procedure of radical cholecystectomy (including atypical resection of liver segments IVb-V) 
Table 2. Pre- and postoperative tumour characteristics

\begin{tabular}{lll}
\hline Incidental GBC (3 patients) & & Suspected GBC (5 patients) \\
\hline $\begin{array}{l}\text { Preoperative tumour size } \\
\text { No evidence of liver mass }\end{array}$ & & $19.6 \mathrm{~mm}$ (range $12-31 \mathrm{~mm})$ \\
$\begin{array}{l}\text { Preoperative T stage } \\
\text { T1b }\end{array}$ & $2 / 3(66.6 \%)$ & $1 / 5(20 \%)$ \\
T2a & $1 / 3(33.3 \%)$ & $0 / 5(0 \%)$ \\
T2b & $0 / 3(0 \%)$ & $2 / 5(40 \%)$ \\
T3 & $0 / 3(0 \%)$ & $2 / 5(40 \%)$ \\
Postoperative T stage & & $1 / 5(20 \%)$ \\
T1b & $2 / 3(66.6 \%)$ & $0 / 5(0 \%)$ \\
T2a & $1 / 3(33.3 \%)$ & $1 / 5(20 \%)$ \\
T2b & $0 / 3(0 \%)$ & $3 / 5(60 \%)$ \\
T3 & $0 / 3(0 \%)$ & \\
Lymph node status & & $4 / 5(80 \%)$ \\
N0 & $2 / 3(66.6 \%)$ & $1 / 5(20 \%)$ \\
N+ & $1 / 3(33.3 \%)$ & \\
Total lymph nodes & & $0 / 5(0 \%)$ \\
1-6 & $0 / 3(0 \%)$ & $1 / 5(20 \%)$ \\
$7-12$ & $1 / 3(33.3 \%)$ & $4 / 5(80 \%)$ \\
$>12$ & $2 / 3(66.6 \%)$ & $25.75 \pm 2.25$ \\
Mean & $22.35 \pm 1.75$ & \\
\hline
\end{tabular}

GBC: gallbladder cancer

or open surgery. In one case, minor intraoperative bleeding from the inferior vena cava occurred during lymphadenectomy and was effectively managed by robotic suturing with prolene $3 / 0$ stiches. Operative time was $46.25 \mathrm{~min}$ (range 30-70 $\mathrm{min}$ ) for robot docking time and adhesiolysis (5 patients having extensive adhesions due to previous laparotomic abdominal surgery) and $147.5 \mathrm{~min}$ (range 110-220 min) for robotic accomplishment of radical cholecystectomy. Mean blood loss was $198.75 \mathrm{~mL}$ (range 50-600 mL) and no intraoperative transfusions were needed. No bile leaks nor lymphatic fistula occurred. Mean postoperative stay was 6 days (range 5-11 days). Postoperative morbidity included one postoperative bleeding treated with a transfusion of packed red blood cell (Clavien-Dindo grade II complication) and one re-intervention for a strangulated bowel loop herniated at one of the trocar site (Clavien-Dindo grade IIIb complication). All patients had an Ro liver resection and 2 patients had N positive disease at final pathology. Mean lymph nodes yield was $25.75 \pm 2.25$, and all patients had more than 6 retrieved lymph nodes. Intraoperative and postoperative information is provided in Table 3. The cystic duct margin was negative in all patients as well as the station 16 sampling for frozen section analysis. With a mean follow-up of 17.5 months (range 29.37.3 months), all patients are alive and all but one, who experienced a peritoneal recurrence and is currently undergoing chemotherapy, are free from disease and under clinical follow-up. No port site metastases were observed during the follow-up period.

\section{DISCUSSION}

The current study is one of the very few reported series on the robotic approach to the surgical treatment of GBC. We presented a prospective series of eight consecutive patients operated for GBC by a robotic approach with results comparable to those reported in the recent literature and showed the feasibility and the safety of this minimally invasive approach. Despite that cholecystectomy was the first surgical intervention widely performed by laparoscopy, a strong reluctance accompanied the adoption of the minimally invasive approach for the treatment of GBC, which is one of the most aggressive cancers of the biliary tract and is generally associated with a poor prognosis. One of the major concerns related to the adoption of the minimally invasive approach for GBC has been the fear of port site recurrence, which has been historically reported to occur in up to $18.6 \%$ of cases in the case of incidental $\mathrm{GBC}^{[16]}$. Tumour cell implantation at the port sites is postulated to occur by extraction of surgical specimen without protective 
Table 3. Intraoperative and postoperative information

\begin{tabular}{ll}
\hline Robot docking time + adhesiolysis & $\mathbf{4 6 . 2 5} \mathbf{~ m i n ~ ( r a n g e ~ 3 0 - 7 0 )}$ \\
\hline Robotic radical cholecystectomy operative time & $147.5 \mathrm{~min}$ (range 110-220) \\
$\begin{array}{l}\text { Intraoperative blood loss } \\
\text { Intraoperative complications }\end{array}$ & $198.75 \mathrm{~mL}$ (range 50-600) \\
$\begin{array}{l}\text { Bleeding } \\
\text { Conversion rate }\end{array}$ & $1 / 8(12.5 \%)$ \\
Postoperative complications & $0 / 8(0 \%)$ \\
Bleeding & $1 / 8(12.5 \%)$ \\
Ventral hernia on port defect & $1 / 8(12.5 \%)$ \\
Hospital stay & 6 days (range 5-11) \\
Follow-up & \\
Mean & 17.5 months (range 29.3-7.3) \\
\hline
\end{tabular}

bag, contact with contaminated instruments (especially in case of bile spillage) or by nidation of exfoliated tumour cells brought to the port site by a sort of aerosol effect created by desufflation of the pneumoperitoneum. The historically reported data are perhaps related to an inappropriate surgical technique carried out in the early years of the learning curve of laparoscopic cholecystectomy for presumed benign disease and were probably associated with gallbladder perforation, bile spillage and no use of protective bag for specimen extraction. The key role of bile spillage during index cholecystectomy for incidentally diagnosed GBC has been addressed in a population-based study by Horkoff et al. ${ }^{[17]}$ who highlighted in a retrospective cohort comparison the negative prognostic impact of bile spillage and its role in the development of peritoneal carcinomatosis. The occurrence of incidentally diagnosed GBC after cholecystectomy is assumed to vary between $0.19 \%$ and $3.3 \%{ }^{[18]}$ with a slight increase after the advent of laparoscopic cholecystectomy. Generally, simple cholecystectomy is considered an adequate treatment for Tis and T1a cancers while a re-intervention consisting in a radical cholecystectomy as first described by Glenn and Hays ${ }^{[19]}$ (which includes locoregional lymphadenectomy and gallbladder bed liver resection) is suggested to resect any potential residual disease and obtain an adequate staging. Since cholecystectomy has already been performed, revision surgery for incidentally diagnosed GBC is not at risk of tumour seeding associated with bile spillage. Full-thickness resection of the port insertion sites at index cholecystectomy has been advocated to minimize the incidence of port site recurrence ${ }^{[20]}$, but as demonstrated by Maker et al. ${ }^{[21]}$ port site resection is not associated with improved survival or disease recurrence and should not be considered mandatory. Nevertheless, radical revision surgery can be very technically demanding because of the presence of fibrosis and inflammatory adhesions often present in the gallbladder bed and at the hepatodudoenal ligament, thus complicating the identification of the vasculobiliary structures and the risk of bile duct injury during radical lymphadenectomy. Only in the last decade, some authors have advocated the minimally invasive surgical treatment of clinically suspected or incidentally diagnosed GBC, highlighting the feasibility and apparent safety of this approach in terms of oncologic outcomes. In 2011 , Belli et al. ${ }^{[8]}$ published their initial series of patients with incidental GBC who underwent a revision procedure by a totally laparoscopic approach, reporting satisfactory clinical outcomes in terms of perioperative and middle term oncologic results. Recently, Vega et al. ${ }^{[9]}$ reported the results of a multicentre retrospective study of patients with incidental GBC who underwent re-resection with curative intent at four centres (including 65 patients operated by a laparoscopic approach) and concluded that a laparoscopic approach for radical re-resection has similar morbidity and oncologic outcomes as open radical re-resection. Feng et al. ${ }^{[10]}$ conducted a comparative analysis of open (61 patients) versus laparoscopic (41 patients) cholecystectomy and radical cholecystectomy for Tis-T3 GBC and found no differences between the two approaches in terms of lymph node retrieval and survival outcomes. Similar results were reported in the retrospective comparative series (open $v$ s. laparoscopic approach) published by Jang et al. ${ }^{[11]}$ and Dou et al. ${ }^{[12]}$ In the study by Agarwal et al. ${ }^{[13]}$, also analysed in a retrospective comparative design were the outcomes of GBC patients (with limited liver infiltration or incidental diagnosis) who underwent laparoscopic radical resection versus those of patients who underwent open radical 
cholecystectomy during the same period. They concluded that laparoscopic radical cholecystectomy is safe and feasible in selected patients with GBC and can offer similar results as open approach. An expert consensus statement published in 2019 recognises that the laparoscopic approach to GBC seems to have favourable outcomes in selected cases operated by expert teams ${ }^{[22]}$ but also highlights that the minimally invasive approach for GBC is still in the early phase of the adoption curve, and more data are needed to assess the outcomes of the procedure. Only anecdotal reports on the surgical treatment of GBC by a robotic approach are currently available in the literature. Byun et al. ${ }^{[23]}$ described their robotic technique for the resection of 13 patients with $\mathrm{T} 2$ or greater stage GBC and highlighted the feasibility and safety of the robotic approach and adequate lymph node retrieval. Goel et al. ${ }^{[24]}$ compared the operative outcomes of 23 patients submitted to robotic radical cholecystectomy to those of 70 patients submitted to open procedure and reported a $14.8 \%$ conversion rate and equivalent oncologic and perioperative outcomes between the two approaches. There is no consensus on the extent of optimal lymphadenectomy for GBC, but in the authors' opinion, a full locoregional node clearance including retro-pancreatic nodes should be performed together with interaortocaval sampling (station 16b1). In fact, as demonstrated by Agarwal et al. ${ }^{[25]}$, routine sampling at this level prevents non-therapeutic radical resection in $18.6 \%$ of patients deemed resectable on preoperative imaging and staging laparoscopy. The minimum number of harvested nodes for GBC is still a matter of debate, where the 8 th edition of the $\mathrm{AJCC}^{[26]}$ recommends a cut-off of six retrieved nodes for GBC. In our series, all patients had more than 6 lymph nodes retrieved, which is in line with the results of reported open series and fulfil the benchmarks proposed by the AJCC. Radical extended cholecystectomy or radicalisation of incidentally GBC can be technically demanding procedures consisting in a liver resection and an accurate lymphadenectomy, including the retropancreatic nodes and a full clearance of the hepatoduodenal ligament, which requires a high grade of dexterity when performed by unidirectional instruments as in laparoscopy. Appropriate lymphadenectomy can be performed safely and effectively by laparoscopy as demonstrated by Ratti et al. ${ }^{[27]}$, but it deserves advanced laparoscopic skills and a suitable learning curve. In our opinion, the application of the robotic platform in this settings, thanks to higher dexterity achievable with the robotic instruments, which with the endowrist system have seven degrees of freedom, can facilitate adequate surgical manipulation and the achievement of an appropriate lymph node clearance in a confined space such as the hepatic pedicle. The magnified high-resolution 3D stereoscopic view offered by the robotic platform is also an added value in defining the anatomical structures. As regards to the extent of liver resection for GBC, there is still no broad consensus, and parenchymal resection is generally tailored on $\mathrm{T}$ stage and tumour size and location ${ }^{[28,29]}$. While for T1 $\mathrm{b}$ and $\mathrm{T} 2$ cancers, an extended cholecystectomy (atypical resection of segment IVb-V or a formal bisegmentectomy) is generally considered adequate, but the optimal extent of liver resection for T3 tumours is still unclear. Since GBC is staged as T3 by the AJCC for any infiltration of liver parenchyma, regardless of the location and size of the tumour, the surgical treatment can vary widely from an ultrasound-guided atypical resection (for liver bed type tumours located at the gallbladder fundus) to up a formal extended hemi-hepatectomy (generally reserved for T3 gallbladder neck and hepatic hilum type tumours). In our series, we decided on a robotic approach only in patients with liver bed type lesions of the gallbladder fundus and a limited liver involvement [mean tumour size of $19.6 \mathrm{~mm}$ (range $12-31 \mathrm{~mm}$ )], and therefore we considered appropriate an atypical resection of segments $\mathrm{IVb}-\mathrm{V}$ with a an ultrasound-checked free margin of at least $2-3 \mathrm{~cm}^{[30]}$. Such as for laparoscopy, the robotic approach when compared to the standard open approach, which requires a wide bilateral subcostal incision, can promote a faster recovery, as demonstrated by the short postoperative stay of our series, and a fast access to adjuvant chemotherapy when appropriate. This an important issue in a biologically aggressive disease such as GBC and could play a role in prolonging survival. As regards to early oncologic outcomes, no port site metastases were observed during the followup period in the current series. One of our patients experienced an early peritoneal recurrence, but this is more likely to be related to the advanced stage of the disease ( $\left.\mathrm{T}_{2} \mathrm{~N} 1\right)$ and to the adenosquamous histological type of the cancer than to any factor related to the surgical approach. This is one of the very few reports currently available in the literature on the robotic treatment of GBC. Our series is limited and 
needs further evaluation especially in terms of oncologic outcomes, but we did demonstrate the safety and technical feasibility of the robotic approach for the treatment of selected GBC. With an accurate patient selection, a low threshold for conversion and a proper surgical technique which includes careful tissue manipulation, the avoidance of any bile spillage, an adequate locoregional lymphadenectomy and the use of a protective plastic bag for specimen extraction, similar outcomes as with open surgery can be offered by a robotic approach. In addition, the use of the robotic platform has the potential to facilitate the surgical procedure when compared to laparoscopy, speed up the learning curve and maintain the benefits of better short-term outcomes and rapid recovery associated with minimally invasive surgery.

In conclusion, our study described a standardized step-by-step robotic technique for the surgical treatment of clinically suspected or incidentally diagnosed GBC and demonstrated the feasibility and the safety of the robotic approach in this setting. More data and multicentre series are needed to confirm our results and to assess the oncologic outcomes of the robotic approach.

\section{DECLARATIONS}

\section{Acknowledgments}

Thanks go to Assunta Zazzaro for her appreciated work and team coordination.

\section{Authors' contributions}

Conception and design: Belli A, Patrone R, Izzo F

Manuscript writing, collection and assembly of data, data analysis and interpretation: Belli A, Patrone R

Provision of study materials or patients, critical revision and final approval of manuscript: Belli A, Patrone R, Albino V, Leongito M, Piccirillo M, Granata V, Pasta G, Palaia R, Izzo F

\section{Availability of data and materials}

Data are stored in a database at Fondazione G. Pascale IRCCS Naples.

\section{Financial support and sponsorship}

None.

\section{Conflicts of interest}

All authors declared that there are no conflicts of interest.

\section{Ethical approval and consent to participate}

The study was approved by the local ethics committee. The informed consent to participate of patients was obtained.

\section{Consent for publication}

The informed consent for publication of patients was obtained.

\section{Copyright}

(c) The Author(s) 2020.

\section{REFERENCES}

1. Deijen CL, Vasmel JE, de Lange-de Klerk ESM, et al. COLOR (Colon cancer Laparoscopic or Open Resection) study group. Ten-year outcomes of a randomised trial of laparoscopic versus open surgery for colon cancer. Surg Endosc 2017;31:2607-15.

2. Kang SB, Park JW, Jeong SY, et al. Open versus laparoscopic surgery for mid or low rectal cancer after neoadjuvant chemoradiotherapy (COREAN trial): short-term outcomes of an open-label randomised controlled trial. Lancet Oncol 2010;11:637-45.

3. Yu J, Huang C, Sun Y, et al. Chinese Laparoscopic Gastrointestinal Surgery Study (CLASS) Group. Effect of laparoscopic vs open distal gastrectomy on 3-year disease-free survival in patients with locally advanced gastric cancer: the CLASS-01 randomized clinical trial. 
JAMA 2019;321:1983-92.

4. Syn NL, Kabir T, Koh YX, et al. Survival advantage of laparoscopic versus open resection for colorectal liver metastases: a meta-analysis of individual patient data from randomized trials and propensity-score matched studies. Ann Surg 2020;272:253-65.

5. Fretland ÅA, Dagenborg VJ, Bjørnelv GMW, et al. Laparoscopic versus open resection for colorectal liver metastases: the OSLOCOMET randomized controlled trial. Ann Surg 2018;267:199-207.

6. Morise Z, Aldrighetti L, Belli G, et al. ILLS-Tokyo Collaborator group. Laparoscopic repeat liver resection for hepatocellular carcinoma: a multicentre propensity score-based study. Br J Surg 2020;107:889-95.

7. Belli G, Fantini C, Belli A, Limongelli P. Laparoscopic liver resection for hepatocellular carcinoma in cirrhosis: long-term outcomes. Dig Surg 2011;28:134-40.

8. Belli G, Cioffi L, D’Agostino A, et al. Revision surgery for incidentally detected early gallbladder cancer in laparoscopic era. $J$ Laparoendosc Adv Surg Tech A 2011;21:531-4.

9. Vega EA, De Aretxabala X, Qiao W, et al. Comparison of oncological outcomes after open and laparoscopic re-resection of incidental gallbladder cancer. Br J Surg 2020;107:289-300.

10. Feng JW, Yang XH, Liu CW, et al. Comparison of laparoscopic and open approach in treating gallbladder cancer. J Surg Res 2019;234:269-76.

11. Jang JY, Han HS, Yoon YS, Cho JY, Choi Y. Retrospective comparison of outcomes of laparoscopic and open surgery for T2 gallbladder cancer - Thirteen-year experience. Surg Oncol 2019;29:142-7.

12. Dou C, Zhang Y, Liu J, et al. Laparoscopy versus laparotomy approach of a radical resection for gallbladder cancer: a retrospective comparative study. Surg Endosc 2020;34:2926-38.

13. Agarwal AK, Javed A, Kalayarasan R, Sakhuja P. Minimally invasive versus the conventional open surgical approach of a radical cholecystectomy for gallbladder cancer: a retrospective comparative study. HPB (Oxford) 2015;17:536-41.

14. Dindo D, Demartines N, Clavien PA. Classification of surgical complications: a new proposal with evaluation in a cohort of 6336 patients and results of a survey. Ann Surg 2004;240:205-13.

15. Koch M, Garden OJ, Padbury R, et al. Bile leakage after hepatobiliary and pancreatic surgery: a definition and grading of severity by the International Study Group of Liver Surgery. Surgery 2011;149:680-8.

16. Berger-Richardson D, Chesney TR, Englesakis M, et al. Trends in port-site metastasis after laparoscopic resection of incidental gallbladder cancer: a systematic review. Surgery 2017;161:618-27.

17. Horkoff MJ, Ahmed Z, Xu Y, et al. Adverse outcomes after bile spillage in incidental gallbladder cancers: a population-based study. Ann Surg 2019; doi: 10.1097/SLA.0000000000003325.

18. Søreide K, Guest RV, Harrison EM, et al. Systematic review of management of incidental gallbladder cancer after cholecystectomy. $B r J$ Surg 2019;106:32-45.

19. Glenn F, Hays DM. The scope of radical surgery in the treatment of malignant tumors of the extrahepatic biliary tract. Surg Gynecol Obstet 1954;99:529-41.

20. Giuliante F, Ardito F, Vellone M, Clemente G, Nuzzo G. Port-sites excision for gallbladder cancer incidentally found after laparoscopic cholecystectomy. Am J Surg 2006;191:114-6.

21. Maker AV, Butte JM, Oxenberg J, et al. Is port site resection necessary in the surgical management of gallbladder cancer? Ann Surg Oncol 2012;19:409-17.

22. Han HS, Yoon YS, Agarwal AK, et al. Laparoscopic surgery for gallbladder cancer: an expert consensus statement. Dig Surg 2019;36:1-6.

23. Byun Y, Choi YJ, Kang JS, et al. Robotic extended cholecystectomy in gallbladder cancer. Surg Endosc 2020;34:3256-61.

24. Goel M, Khobragade K, Patkar S, Kanetkar A, Kurunkar S. Robotic surgery for gallbladder cancer: operative technique and early outcomes. J Surg Oncol 2019;119:958-63.

25. Agarwal AK, Kalayarasan R, Javed A, Sakhuja P. Role of routine $16 \mathrm{~b} 1$ lymph node biopsy in the management of gallbladder cancer: an analysis. HPB (Oxford) 2014;16:229-34.

26. Zhu AX, Pawlik TM, Kooby DA, Schefter TE, Vauthey JN. Gallbladder AJCC Cancer Staging Manual (8th edn). New York: Springer International; 2017.

27. Ratti F, Fiorentini G, Cipriani F, et al. Perioperative and long-term outcomes of laparoscopic versus open lymphadenectomy for biliary tumors: a propensity-score-based, case-matched analysis. Ann Surg Oncol 2019;26:564-75.

28. Lee SE, Kim KS, Kim WB, et al; Korean Association of Hepato-Biliary and Pancreas Surgery. Practical guidelines for the surgical treatment of gallbladder cancer. J Korean Med Sci 2014;29:1333-40.

29. Pilgrim C, Usatoff V, Evans PM. A review of the surgical strategies for the management of gallbladder carcinoma based on T stage and growth type of the tumour. Eur J Surg Oncol 2009;35:903-7.

30. Kapoor VK. Advanced gallbladder cancer: Indian “middle path”. J Hepatobiliary Pancreat Surg 2007;14:366-73. 